\title{
In Silico Analysis of Biomarker Potentials of miRNA- Mediated ceRNAs in Gastric Neoplasms
}

\author{
Diler Us Altay ${ }^{1}$, Sercan Ergün ${ }^{2}$ \\ ${ }^{1}$ Department of Nutrition and Dietetics, Faculty of health sciences, Ordu University, Ordu, Turkey \\ ${ }^{2}$ Department of Medical Biology, Faculty of Medicine, Ordu University, Ordu, Turkey
}

Received: 27 May 2019, Accepted: 2 July 2019, Published online: 28 August 2019

(C) Ordu University Institute of Health Sciences, Turkey, 2019

\begin{abstract}
Objectives: The objective of this study is to define novel biomarkers for gastric neoplasm (GN) via in silico analysis that takes GN-specific miRNAs, finds their combinatorial target genes (potential ceRNAs), selects ones containing T-UCR among them and potentiates their relevance with GN. Based on this study we can plan new in vitro and in vivo studies.
\end{abstract}

Methods: Four miRNAs of which clinical relevances with GN were proved experimentally were exported via mirTarbase. Using the ComiR database, 1008 genes targeted by these 4 miRNAs simultaneously were identified. Genes containing T-UCR and showing potential ceRNA activity were extracted. Among GNassociated ceRNAs including T-UCR, we identified genes with significant expression differences between GN and normal stomach tissue using the GEPIA database. The statistical evaluation of the association of NFAT5 and CLK3 genes with GN was performed by Spearman correlation test in GEPIA database.

Results: GN-associated ceRNAs cross-matching with genes including T-UCR in their exonic regions were NFAT5 and CLK3. We identified genes with significant expression differences between GN and normal stomach tissues among GN-associated ceRNAs including T-UCR. According to this analysis, only NFAT5 gene was significantly higher expressed in GN than in normal stomach tissue while the other didn't show any significant differential expression pattern. NFAT5 and CLK3 genes were found to be significantly correlated with GN ( $\mathrm{p}<0.001 ; \mathrm{R}=0.22)$

Conclusion: All in all, this is the study associating NFAT5 gene with GN for the first time and giving it ongogenic potential for GN. Still, larger and more comprehensive studies are needed on this issue.

Key words: Gastric neoplasms; miRNA; ceRNA; T-UCR; In silico analysis

Suggested Citation: Us Altay D, Ergun S. In Silico Analysis of Biomarker Potentials of miRNA-Mediated ceRNAs in Gastric Neoplasms. Middle Black Sea Journal of Health Science, 2019; 5(2):106-119.

\section{Address for correspondence/reprints:}

Diler Us Altay

Telephone number: +90 (452) 8616427

E-mail: surelid@hotmail.com

DOI: $10.19127 / \mathrm{mbsjohs.570444}$

\section{Introduction}

Gastric neoplasms may manifest in various different forms, depending on the cell of origin. The most common form is adenocarcinoma, while lymphoma, gastrointestinal stromal tumors (GISTs), carcinoids and other neoplasms are less frequently seen. Gastric adenocarcinoma is a particularly common cancer across the world, but particularly in the Far East. A lower incidence has been reported in the United Kingdom, but poor prognosis when the disease is in the late stage results in a significant impact on population health. Advanced disease is observed in the majority of patients at time of diagnosis (Schiller, 2017). 
MicroRNAs (miRNAs) are small RNAs that are not encode a protein, but nevertheless potent coordinating capacities. They perform vital regulatory functions in a range of malign cancers, involving gastric cancer. Abnormal stated miRNAs are also involved in gastric carcinogenesis through modification of growth of cells, cell cycles, apoptosis, and migration of cells. Epigenetic and genetic alteration has been identified as one of the mechanisms responsible for miRNA dysregulation. MiRNA performs essential functions in the progression of gastric cancer by targeting oncogene or tumor suppressor gene expression. The first step in determining the roles of miRNAs in gastric cancer is to investigate differences in miRNA expression profiles between normal and tumor gastric tissues (Pan et al., 2013). Tumor suppressor miRNAs inhibit tumor formation by suppressing oncogenes. Relationship of microRNAs within cancer changes proteinencoding oncogene or tumor suppressor genes are known to cause cancer. Genetic cause of cancer with the recent demonstration of miRNAs in tumor formation.

Competing endogenous RNAs (ceRNAs) are transcripts capable of mutual regulation at the posttranscription level through competition for shared miRNAs. CeRNA networks link protein-coding mRNA functions with those of non-coding RNAs (ncRNAs), including microRNA, long ncRNA, pseudogenic RNA and circular RNA. Since any transcripts containing an miRNA response component are in theory capable of acting as ceRNAs, these may represent a widespread form of post-transcriptional gene expression regulation in physiological and pathological terms. A number of factors are known to be capable of affecting ceRNA activity, including the abundance and subcellular localization of ceRNA components, the binding affinity of miRNAs to their sponges, RNA editing, RNA secondary structures and RNAbinding proteins. Disturbance in these may lead to deregulation of ceRNA networks and thus to human diseases, including cancer (Qi et al., 2015).

In recent years, ncRNAs have generated considerable interest in terms of cell transformation. Ultraconserved regions (UCRs) were first discovered in 2004 following bioinformatic investigation of mouse, rat, and human genomes. UCRs consist of a minimum of 481 genomic sequences at least $200 \mathrm{bp}$ in length (range 200-779 bp), and which are fully conserved (100\% identity without any insertions or deletions) among the above three vertebrate species. A significant proportion of UCRs are transcribed (TUCRs) in normal human tissues, and their expression levels have been observed to exhibit a ubiquitous and tissue-specific pattern. While the functions of T-UCRs are largely unclear, the high level of trans-species conservation they exhibit appears to suggest that they are of significant importance to ontogenesis/phylogenesis in mammals. Recent research into genome-wide expression has revealed that T-UCRs exhibit distinct profiles in different human cancers, representing further evidence of their role in carcinogenesis in humans (Fassan et al., 2014).

In recent years understanding their role in cancer, miRNAs have been hopeful in understanding the molecular pathology of cancer and developing molecular targeted therapies. Based on this feature of miRNAs, we aim to identify genes with potential oncogenic activity not previously identified in silico in gastric cancer. In line with our data, we aim to conduct further in vitro and in vivo studies on these miRNAs

\section{Methods}

Selection of miRNAs involved in the pathogenesis of gastric neoplasms

Four miRNAs clinically associated with gastric neoplasm and authenticated experimentally were exported over the MiRTarBase database. The miRTarBase database submits estimated and verified data concerning miRNA-target interaction. This enables researchers to confirm novel miRNA targets. The 'Comfirmed Target module' showed in this study by Chou et al. (2018).

\section{Analysis of gastric neoplasm-specific miRNA- mediated ceRNAs}

One thousand eight genes projected by these four miRNAs simultaneously were described using the ComiR database. ComiR is an online system employed for the purpose of combinatorial miRNA target estimation. It computes the potency of targeting by a group of miRNAs. When calculating the relay impact of one mRNA from a group of several miRNAs, the application employs utilizerdefined miRNA expression levels in a combinatorial manner based on appropriate machine learning techniques and thermodynamic modeling to elicit more accurate estimates. ComiR admits the opportunity of constituting a operational target for a group of miRNAs, based on relevant miRNA expression levels, for every gene (Coronnello and Benos, 2013). 
We hope that the RNA transcripts of these genes will exhibit potential ceRNA activity for these miRNAs and that their arrangement is organized on the basis of miRNA-sponging mechanisms.

Matching of GN-associated ceRNA with genes including T-UCR

Bejerano et al. identified the UCRs in the human genome. Genes including these regions are graded as upstream, exonic or downstream in accordance, depending on the site of fixation within the gene (Bejerano et al., 2004). Genes with T-UCR in their exonic areas were also identified, and those exhibiting latent ceRNA activity were excerpted in our previous research.

Analysis of gastric neoplasm-related ceRNAs including T-UCR in the sense of differential gene expression between gastric neoplasm and normal gastric tissues

Genes exhibiting significant expression differences between gastric neoplasm and normal stomach tissue from GN-associated ceRNAs, including T-UCR were identified with the assistance of the GEPIA database. GEPIA (Gene Expression Profiling Interactive Analysis), a webbased tool to deliver fast and customizable functionalities based on The Cancer Genome Atlas (TCGA) and The Genotype-Tissue Expression (GTEx) data. All plotting features in GEPIA are developed using $\mathrm{R}$ (version 3.3.2) and Perl (version 5.22.1) programs. (Tang et al., 2017).

\section{Correl tests of NFAT5 and CLK3 genes in gastric neoplasm}

Methods for analyzing gene expression are numerous and diverse. Expression-based clustering, for example, can be divided into supervised and unsupervised methods. Gene expression differential analysis is a classical supervised method, leading to the finding tumorspecific genes by comparing tumor to normal groups. Statistical analysis of the association between NFAT5 and CLK3 genes and gastric neoplasm was showed using the Spearman correlation test in the GEPIA database.

\section{Results}

A list of four miRNAs experimentally linked to gastric neoplasm using the miRTarbase database is shown in Table 1.
Table 1. List of miRNAs taking role in gastric neoplasms pathogenesis
1. hsa-miR-148a
2. hsa-miR-23a
3. hsa-miR-370
4. hsa-miR-429

A list of 1008 genes simultaneously targeted by these four miRNAs is shown supplementary 1 . Wedeclared genes with T-UCR in their exonic regions from those listed by Bejerano et al., and these are shown in supplementary 2. Then, we extracted ones showing potential ceRNA activity in our previous analysis among them (Table 2). Genes exhibiting significant expression variation between gastric neoplasm and normal gastric tissues among gastric neoplasm-related ceRNAs with T-UCR were identified. In agreement with that analysis, expression of NFAT5 was significantly higher in gastric neoplasm compared to normal stomach tissue, while no significant differential expression patterns were detected in the other genes (Table 3).

Table 2. List of gastric neoplasms-associated ceRNAs cross-matching with genes including TUCR in their exonic regions

\section{NFAT5 \\ CLK3}

Table 3. Expression values of $\mathrm{GN}$-associated ceRNAs including T-UCR between gastric neoplasms and normal stomach tissues.

\begin{tabular}{lll}
\hline Gene ID & GN & Normal stomach \\
\hline NFAT5* & $\mathbf{8 , 9 4}$ & $\mathbf{4 , 0 3}$ \\
\hline$C L K 3$ & 30,1 & 36,53
\end{tabular}

*shows significantly differential expression pattern between GN and normal stomach tissues

Statistical analysis of the link between NFAT5 and CLK3 genes and gastric neoplasm was conducted through the GEPIA database. Spearman correlation analysis revealed that the NFAT5 and CLK3 gene pair exhibited significant association with gastric neoplasm (Figure 1) $\quad(p=0.000$; $\mathrm{R}=0.22$ ). 


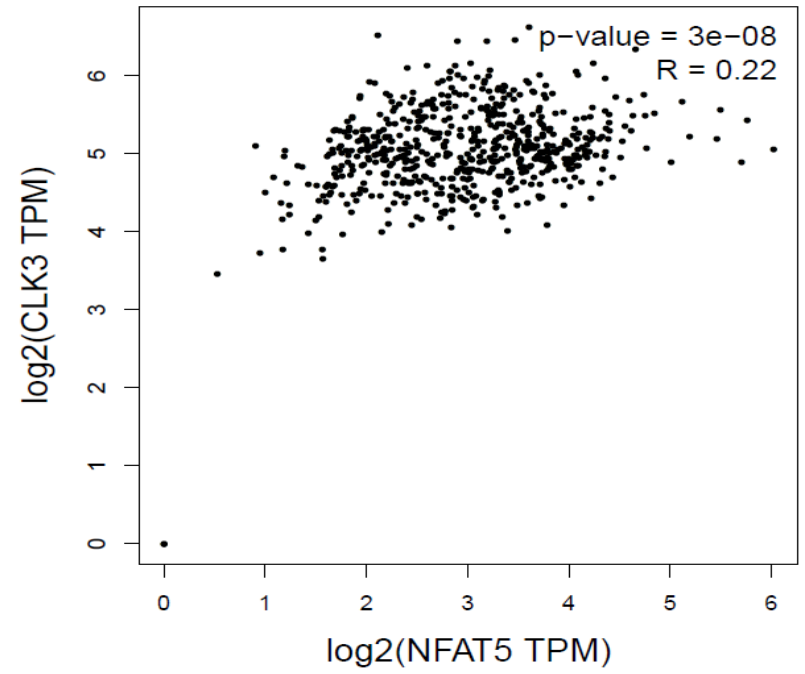

Figure 1: Spearman correlation analysis of NFAT5 and CLK3 genes with GN.

Supplementary 1. List of genes targeted bu these 4GN-associated miRNAs simultaneously

\begin{tabular}{|c|c|}
\hline AVPR1A & 0.9048 \\
\hline AAK1 & 0.9202 \\
\hline $\mathrm{ABCC} 3$ & 0.9049 \\
\hline $\mathrm{ABI} 2$ & 0.9239 \\
\hline ACADSB & 0.9122 \\
\hline ACER3 & 0.905 \\
\hline ACP6 & 0.9129 \\
\hline ACSS3 & 0.9052 \\
\hline ACVR1C & 0.9173 \\
\hline ACVR2B & 0.9216 \\
\hline ADAM10 & 0.9201 \\
\hline ADAM12 & 0.9212 \\
\hline ADAM22 & 0.9054 \\
\hline ADAMTS4 & 0.9054 \\
\hline ADAMTS5 & 0.9197 \\
\hline ADAMTS6 & 0.9213 \\
\hline ADARB1 & 0.9051 \\
\hline ADCY1 & 0.9202 \\
\hline ADCYAP1R1 & 0.9128 \\
\hline ADRBK2 & 0.9055 \\
\hline AFF1 & 0.9197 \\
\hline AFF2 & 0.9133 \\
\hline AFF3 & 0.9051 \\
\hline AGAP1 & 0.923 \\
\hline AGFG1 & 0.9172 \\
\hline AGO1 & 0.9217 \\
\hline $\mathrm{AGO} 2$ & 0.9116 \\
\hline AGO3 & 0.9231 \\
\hline AJAP1 & 0.9201 \\
\hline
\end{tabular}

\begin{tabular}{|c|c|}
\hline AK4 & 0.9053 \\
\hline AKAP5 & 0.9172 \\
\hline AKAP6 & 0.9054 \\
\hline AKT2 & 0.9114 \\
\hline AMER2 & 0.9131 \\
\hline ANK2 & 0.9172 \\
\hline ANKFY1 & 0.9125 \\
\hline ANKRD11 & 0.9201 \\
\hline ANKRD9 & 0.9049 \\
\hline ANO5 & 0.9111 \\
\hline ANTXR2 & 0.9131 \\
\hline AP1M1 & 0.9178 \\
\hline AP5M1 & 0.9201 \\
\hline $\mathrm{APC}$ & 0.9052 \\
\hline APOL3 & 0.9104 \\
\hline APOL6 & 0.9055 \\
\hline ARHGAP19 & 0.9051 \\
\hline ARHGAP20 & 0.9174 \\
\hline ARHGAP31 & 0.9049 \\
\hline ARHGAP32 & 0.9052 \\
\hline ARIH1 & 0.9227 \\
\hline ARL10 & 0.9134 \\
\hline ARL5A & 0.9167 \\
\hline ARL8B & 0.9146 \\
\hline ARNT2 & 0.9213 \\
\hline ARPIN & 0.9174 \\
\hline ARSD & 0.9117 \\
\hline ASAP1 & 0.9165 \\
\hline ASRGL1 & 0.909 \\
\hline
\end{tabular}

\begin{tabular}{|c|c|}
\hline ATF7 & 0.9127 \\
\hline ATG9A & 0.9127 \\
\hline ATP10A & 0.9051 \\
\hline ATP11A & 0.9198 \\
\hline ATP11B & 0.9117 \\
\hline ATP2A2 & 0.9051 \\
\hline ATP8A1 & 0.9198 \\
\hline ATP8A2 & 0.9054 \\
\hline ATRX & 0.923 \\
\hline ATXN1 & 0.9216 \\
\hline ATXN1L & 0.9054 \\
\hline ATXN3 & 0.9202 \\
\hline ATXN7L3B & 0.9175 \\
\hline B3GALT5 & 0.9201 \\
\hline B4GALT4 & 0.905 \\
\hline B4GALT5 & 0.9163 \\
\hline $\mathrm{BACH} 2$ & 0.913 \\
\hline BAG2 & 0.9125 \\
\hline BCAR1 & 0.9128 \\
\hline BCAS4 & 0.9054 \\
\hline BCL2L11 & 0.9209 \\
\hline BICD1 & 0.9197 \\
\hline BICD2 & 0.905 \\
\hline $\mathrm{BMF}$ & 0.9049 \\
\hline BMP1 & 0.912 \\
\hline BMP2K & 0.9166 \\
\hline BMPR1A & 0.923 \\
\hline $\mathrm{BNC2}$ & 0.9216 \\
\hline BNIP2 & 0.917 \\
\hline
\end{tabular}


Analysis of miRNA-Mediated ceRNAs in Gastric Neoplasms

\begin{tabular}{|c|c|}
\hline BRCA1 & 0.9171 \\
\hline BRWD1 & 0.9173 \\
\hline C12orf49 & 0.9176 \\
\hline C14orf37 & 0.9125 \\
\hline C16orf52 & 0.9168 \\
\hline C17orf51 & 0.92 \\
\hline C17orf70 & 0.9048 \\
\hline C18orf25 & 0.9116 \\
\hline C18orf32 & 0.9195 \\
\hline C1orf95 & 0.9056 \\
\hline C2orf71 & 0.9162 \\
\hline C4orf32 & 0.9132 \\
\hline C5orf56 & 0.9116 \\
\hline C9orf114 & 0.9208 \\
\hline CA12 & 0.9049 \\
\hline CA5B & 0.9051 \\
\hline CAB39L & 0.9113 \\
\hline CACNA1E & 0.9176 \\
\hline CACNG8 & 0.9177 \\
\hline CACUL1 & 0.923 \\
\hline CADM1 & 0.9132 \\
\hline CADM2 & 0.9056 \\
\hline CAMK4 & 0.9201 \\
\hline CAMSAP2 & 0.911 \\
\hline CAPRIN2 & 0.913 \\
\hline CARD8 & 0.905 \\
\hline CASK & 0.913 \\
\hline CASP10 & 0.9166 \\
\hline CBFA2T2 & 0.9052 \\
\hline CBX5 & 0.9177 \\
\hline CCDC127 & 0.9201 \\
\hline CCDC144A & 0.9115 \\
\hline CCDC50 & 0.9175 \\
\hline CCDC85C & 0.9217 \\
\hline CCDC93 & 0.9128 \\
\hline $\mathrm{CCNT} 2$ & 0.9127 \\
\hline CCSAP & 0.9125 \\
\hline CD226 & 0.9055 \\
\hline CD84 & 0.9053 \\
\hline CDCP1 & 0.9117 \\
\hline $\mathrm{CDH} 23$ & 0.9173 \\
\hline $\mathrm{CDH} 7$ & 0.9214 \\
\hline CDH8 & 0.9232 \\
\hline CDHR1 & 0.9211 \\
\hline
\end{tabular}

\begin{tabular}{|c|c|}
\hline CDKL5 & 0.9177 \\
\hline CDS2 & 0.9133 \\
\hline CDYL2 & 0.9174 \\
\hline CECR2 & 0.9173 \\
\hline CELF1 & 0.9225 \\
\hline CELF2 & 0.9132 \\
\hline CENPO & 0.9117 \\
\hline CENPP & 0.9176 \\
\hline CEP192 & 0.9132 \\
\hline CEP250 & 0.9133 \\
\hline CEP78 & 0.913 \\
\hline CEP85L & 0.9207 \\
\hline CERS6 & 0.9125 \\
\hline CFL2 & 0.9232 \\
\hline CFLAR & 0.9057 \\
\hline CHML & 0.9123 \\
\hline CHRM3 & 0.905 \\
\hline CHRNA7 & 0.9167 \\
\hline CHST11 & 0.9127 \\
\hline CHST9 & 0.9056 \\
\hline CIITA & 0.9133 \\
\hline CLCN3 & 0.9104 \\
\hline CLCN6 & 0.912 \\
\hline CLEC16A & 0.905 \\
\hline CLEC2D & 0.9049 \\
\hline CLK3 & 0.9177 \\
\hline CLMN & 0.9134 \\
\hline CLOCK & 0.9201 \\
\hline CLVS2 & 0.9237 \\
\hline CNKSR3 & 0.9238 \\
\hline CNNM2 & 0.9227 \\
\hline CNOT4 & 0.9103 \\
\hline CNOT6L & 0.9228 \\
\hline CNTNAP2 & 0.9049 \\
\hline CNTNAP3B & 0.9119 \\
\hline CNTROB & 0.9121 \\
\hline COL20A1 & 0.9195 \\
\hline CPD & 0.9125 \\
\hline CPEB3 & 0.9053 \\
\hline $\mathrm{CPM}$ & 0.9127 \\
\hline CPSF6 & 0.9127 \\
\hline CRTAP & 0.9198 \\
\hline CSRNP3 & 0.9134 \\
\hline CTNNA3 & 0.9211 \\
\hline
\end{tabular}

\begin{tabular}{|c|c|}
\hline CUL3 & 0.9049 \\
\hline CUX1 & 0.9216 \\
\hline CXCL12 & 0.9162 \\
\hline CXorf23 & 0.9196 \\
\hline CYB561D1 & 0.905 \\
\hline CYB5R4 & 0.9132 \\
\hline CYTH3 & 0.9119 \\
\hline DAPK2 & 0.9175 \\
\hline DBNL & 0.9226 \\
\hline DBT & 0.9133 \\
\hline DCAF7 & 0.9052 \\
\hline $\mathrm{DCN}$ & 0.917 \\
\hline DCP2 & 0.9233 \\
\hline DCUN1D3 & 0.905 \\
\hline DCX & 0.9053 \\
\hline DDI2 & 0.9177 \\
\hline DDX53 & 0.9165 \\
\hline DGKE & 0.9131 \\
\hline DGKH & 0.9227 \\
\hline DGKI & 0.9176 \\
\hline DIRAS2 & 0.9113 \\
\hline DIS3 & 0.9215 \\
\hline DISC1 & 0.9052 \\
\hline DLG5 & 0.9174 \\
\hline DLGAP2 & 0.9055 \\
\hline DNAJC10 & 0.9231 \\
\hline DNAJC15 & 0.9131 \\
\hline DNAJC18 & 0.9167 \\
\hline DNAJC5 & 0.9196 \\
\hline DNASE1 & 0.9177 \\
\hline DNM3 & 0.9127 \\
\hline DNMT3A & 0.9176 \\
\hline DOK6 & 0.9176 \\
\hline DR1 & 0.9216 \\
\hline DRP2 & 0.9122 \\
\hline DSC2 & 0.9215 \\
\hline DTNA & 0.917 \\
\hline DTWD1 & 0.9227 \\
\hline DYRK2 & 0.9174 \\
\hline EEF2K & 0.9051 \\
\hline EGFR & 0.9176 \\
\hline EIF2AK2 & 0.9133 \\
\hline EIF4E3 & 0.9216 \\
\hline EIF4G1 & 0.905 \\
\hline
\end{tabular}


Analysis of miRNA-Mediated ceRNAs in Gastric Neoplasms

\begin{tabular}{|c|c|}
\hline EIF5 & 0.9048 \\
\hline ELFN2 & 0.9129 \\
\hline ELK4 & 0.9174 \\
\hline ELP2 & 0.9052 \\
\hline EMC10 & 0.9056 \\
\hline ENAH & 0.9176 \\
\hline ENTPD1 & 0.9056 \\
\hline EPHA5 & 0.9123 \\
\hline EPHA8 & 0.9106 \\
\hline ЕРНB6 & 0.9122 \\
\hline EPN1 & 0.9217 \\
\hline EPT1 & 0.9053 \\
\hline ERBB2 & 0.9049 \\
\hline ERBB2IP & 0.916 \\
\hline ERBB4 & 0.9175 \\
\hline ERI1 & 0.911 \\
\hline ESRRG & 0.9162 \\
\hline ETNK1 & 0.913 \\
\hline ETV5 & 0.9109 \\
\hline EXOC5 & 0.9052 \\
\hline EXOSC9 & 0.9111 \\
\hline EXT1 & 0.9171 \\
\hline FAM126A & 0.9133 \\
\hline FAM126B & 0.9225 \\
\hline FAM168B & 0.917 \\
\hline FAM179A & 0.9176 \\
\hline FAM193B & 0.9049 \\
\hline FAM204A & 0.9202 \\
\hline FAM217B & 0.9103 \\
\hline FAM26E & 0.9201 \\
\hline FAM63B & 0.92 \\
\hline FAM83F & 0.9178 \\
\hline FAM9C & 0.9209 \\
\hline FARP1 & 0.9175 \\
\hline FARP2 & 0.9126 \\
\hline FAT3 & 0.9198 \\
\hline FBXL4 & 0.9172 \\
\hline FBXO22 & 0.923 \\
\hline FBXO25 & 0.9177 \\
\hline FBXO30 & 0.9174 \\
\hline FBXO32 & 0.9129 \\
\hline FEM1A & 0.9055 \\
\hline FER & 0.9216 \\
\hline FGF14 & 0.9236 \\
\hline
\end{tabular}

\begin{tabular}{|c|c|}
\hline FGF7 & 0.9121 \\
\hline FGFR1OP & 0.9217 \\
\hline FIGN & 0.9215 \\
\hline FILIP1 & 0.9123 \\
\hline FKBP15 & 0.905 \\
\hline FKTN & 0.913 \\
\hline FLNA & 0.9056 \\
\hline FLRT2 & 0.9241 \\
\hline FMN1 & 0.9177 \\
\hline FNTA & 0.9049 \\
\hline FOSL2 & 0.9128 \\
\hline FOXK1 & 0.9177 \\
\hline FOXP2 & 0.913 \\
\hline FREM2 & 0.9052 \\
\hline FRK & 0.9231 \\
\hline FRY & 0.9192 \\
\hline FSD1L & 0.9209 \\
\hline FTO & 0.9056 \\
\hline FUT4 & 0.9224 \\
\hline FUT9 & 0.9233 \\
\hline FXR1 & 0.9175 \\
\hline FZD3 & 0.9134 \\
\hline GAB1 & 0.9051 \\
\hline GABRA4 & 0.9054 \\
\hline GABRG3 & 0.9176 \\
\hline GALR1 & 0.9133 \\
\hline GAN & 0.9217 \\
\hline GAS2L3 & 0.9189 \\
\hline GCC2 & 0.9054 \\
\hline GDAP2 & 0.9199 \\
\hline GDF11 & 0.9056 \\
\hline GFOD1 & 0.9176 \\
\hline GFPT1 & 0.9053 \\
\hline GJA3 & 0.9122 \\
\hline GLRA3 & 0.9173 \\
\hline GMFB & 0.9214 \\
\hline GMPPB & 0.9051 \\
\hline GMPS & 0.905 \\
\hline GNAI3 & 0.9238 \\
\hline GNAO1 & 0.9168 \\
\hline GNB1L & 0.9054 \\
\hline GNB5 & 0.9132 \\
\hline GNPDA2 & 0.9118 \\
\hline GOLGA6L2 & 0.9228 \\
\hline
\end{tabular}

\begin{tabular}{|c|c|}
\hline GOLGB1 & 0.9133 \\
\hline GOLT1B & 0.9106 \\
\hline GPATCH2L & 0.9238 \\
\hline GPR107 & 0.913 \\
\hline GPR161 & 0.9049 \\
\hline GPR180 & 0.9197 \\
\hline GPRC5B & 0.9125 \\
\hline GPRIN3 & 0.9134 \\
\hline GRAMD1B & 0.9049 \\
\hline GREM1 & 0.9227 \\
\hline GRIK3 & 0.9229 \\
\hline GRIN2A & 0.9132 \\
\hline GRIN2B & 0.9241 \\
\hline GTDC1 & 0.9176 \\
\hline GTF2H5 & 0.9132 \\
\hline GTF3C4 & 0.9123 \\
\hline GTPBP10 & 0.9213 \\
\hline GUCY1A2 & 0.9134 \\
\hline GXYLT1 & 0.9197 \\
\hline HDAC2 & 0.9053 \\
\hline HDAC9 & 0.9198 \\
\hline HECW2 & 0.9174 \\
\hline HEG1 & 0.9194 \\
\hline HELB & 0.9176 \\
\hline HELZ & 0.9174 \\
\hline HEMK1 & 0.9231 \\
\hline HFE & 0.9116 \\
\hline HHIP & 0.9051 \\
\hline HIF1AN & 0.9227 \\
\hline HIPK2 & 0.9234 \\
\hline HIPK3 & 0.9123 \\
\hline HLA-A & 0.908 \\
\hline HLA-A & 0.9194 \\
\hline HMGA2 & 0.9053 \\
\hline HMHA1 & 0.9049 \\
\hline HNRNPA3 & 0.9049 \\
\hline HOOK3 & 0.9056 \\
\hline HS2ST1 & 0.9171 \\
\hline HS6ST3 & 0.9131 \\
\hline HSBP1 & 0.9132 \\
\hline HSD17B2 & 0.9055 \\
\hline HSPA12A & 0.9122 \\
\hline HTT & 0.9194 \\
\hline ICA1L & 0.9173 \\
\hline
\end{tabular}


Analysis of miRNA-Mediated ceRNAs in Gastric Neoplasms

\begin{tabular}{|c|c|}
\hline ICE2 & 0.9175 \\
\hline ICOSLG & 0.913 \\
\hline IDS & 0.9127 \\
\hline IFITM10 & 0.9164 \\
\hline IGF2BP1 & 0.9129 \\
\hline IGSF10 & 0.9119 \\
\hline IKZF1 & 0.9125 \\
\hline IL17RD & 0.9054 \\
\hline IL6R & 0.9124 \\
\hline IL6ST & 0.913 \\
\hline ILDR2 & 0.9201 \\
\hline IMPG1 & 0.9125 \\
\hline INO80D & 0.9201 \\
\hline INPP4A & 0.9176 \\
\hline INTS6 & 0.9217 \\
\hline INTU & 0.9133 \\
\hline IPCEF1 & 0.9172 \\
\hline IPMK & 0.9125 \\
\hline IPO9 & 0.9216 \\
\hline IRAK3 & 0.9051 \\
\hline IRGQ & 0.9053 \\
\hline ITGA11 & 0.9172 \\
\hline ITGA9 & 0.9212 \\
\hline ITM2B & 0.9132 \\
\hline ITSN1 & 0.9178 \\
\hline IYD & 0.9213 \\
\hline JAKMIP2 & 0.9168 \\
\hline JMY & 0.905 \\
\hline KALRN & 0.9053 \\
\hline KAT7 & 0.9132 \\
\hline KATNAL1 & 0.913 \\
\hline KCNA1 & 0.9052 \\
\hline KCNB1 & 0.9132 \\
\hline $\mathrm{KCNC1}$ & 0.9052 \\
\hline KCNC4 & 0.9238 \\
\hline KCND3 & 0.9113 \\
\hline KCNH5 & 0.92 \\
\hline KCNJ15 & 0.9131 \\
\hline KCNJ6 & 0.924 \\
\hline KCNK5 & 0.9103 \\
\hline KCNMA1 & 0.9055 \\
\hline KCNN3 & 0.9134 \\
\hline KCNQ3 & 0.9215 \\
\hline KCNQ4 & 0.9099 \\
\hline
\end{tabular}

\begin{tabular}{|c|c|}
\hline KCNQ5 & 0.9119 \\
\hline KCTD15 & 0.9121 \\
\hline KCTD16 & 0.9226 \\
\hline KDM3B & 0.9199 \\
\hline KDM5A & 0.9051 \\
\hline KDM7A & 0.9213 \\
\hline KIAA0930 & 0.9198 \\
\hline KIAA1045 & 0.9124 \\
\hline KIAA1244 & 0.9216 \\
\hline KIAA1456 & 0.9053 \\
\hline KIAA1462 & 0.9171 \\
\hline KIAA1549 & 0.9054 \\
\hline KIAA1614 & 0.9175 \\
\hline KIAA1958 & 0.9175 \\
\hline KIAA2018 & 0.9129 \\
\hline KIDINS220 & 0.9199 \\
\hline KIF1B & 0.9128 \\
\hline KIF26B & 0.9054 \\
\hline KIF6 & 0.9215 \\
\hline KLC1 & 0.9234 \\
\hline KLF12 & 0.9216 \\
\hline KLHL21 & 0.9051 \\
\hline KLHL28 & 0.9116 \\
\hline KLHL42 & 0.905 \\
\hline KLHL6 & 0.9126 \\
\hline KMT2C & 0.9236 \\
\hline KPNA4 & 0.913 \\
\hline KRR1 & 0.9226 \\
\hline KRT222 & 0.9105 \\
\hline KSR1 & 0.9212 \\
\hline KSR2 & 0.9134 \\
\hline KYNU & 0.9236 \\
\hline LANCL3 & 0.9056 \\
\hline LCOR & 0.9054 \\
\hline LCORL & 0.9048 \\
\hline LDLRAD4 & 0.9053 \\
\hline LGALS8 & 0.9127 \\
\hline LPGAT1 & 0.9214 \\
\hline LPHN3 & 0.9131 \\
\hline LPP & 0.9231 \\
\hline LRIG2 & 0.9199 \\
\hline LRRC58 & 0.9197 \\
\hline LRRC8B & 0.9051 \\
\hline LRRK1 & 0.9133 \\
\hline
\end{tabular}

\begin{tabular}{|c|c|}
\hline LRRK2 & 0.9173 \\
\hline LSAMP & 0.9176 \\
\hline LYNX1 & 0.9123 \\
\hline LYRM2 & 0.9126 \\
\hline MACC1 & 0.9171 \\
\hline MAP3K2 & 0.9201 \\
\hline МАР3К9 & 0.9133 \\
\hline MAPK1 & 0.9201 \\
\hline MAPK13 & 0.9052 \\
\hline MAS1 & 0.9056 \\
\hline MBNL3 & 0.9176 \\
\hline MBOAT2 & 0.9054 \\
\hline MBP & 0.9177 \\
\hline $\mathrm{MCC}$ & 0.905 \\
\hline MCFD2 & 0.9205 \\
\hline MCTP2 & 0.9169 \\
\hline MDGA1 & 0.9055 \\
\hline MDM2 & 0.9131 \\
\hline MDM4 & 0.9133 \\
\hline MECP2 & 0.9056 \\
\hline MED12L & 0.9123 \\
\hline MED13L & 0.9201 \\
\hline MEGF9 & 0.919 \\
\hline MEIS1 & 0.9123 \\
\hline MESDC2 & 0.9122 \\
\hline METTL8 & 0.9175 \\
\hline MEX3C & 0.9121 \\
\hline MGAT4A & 0.9131 \\
\hline MGAT4C & 0.9238 \\
\hline MGAT5 & 0.9173 \\
\hline MGLL & 0.9124 \\
\hline MIEF1 & 0.9165 \\
\hline MITF & 0.9114 \\
\hline MKLN1 & 0.923 \\
\hline MLEC & 0.9128 \\
\hline MLXIP & 0.9126 \\
\hline MLYCD & 0.9134 \\
\hline MMP16 & 0.9057 \\
\hline MOB1B & 0.913 \\
\hline MON2 & 0.9131 \\
\hline MOSPD2 & 0.9103 \\
\hline MPP6 & 0.9128 \\
\hline MPRIP & 0.9176 \\
\hline MR1 & 0.9131 \\
\hline
\end{tabular}


Analysis of miRNA-Mediated ceRNAs in Gastric Neoplasms

\begin{tabular}{|c|c|}
\hline MRE11A & 0.9167 \\
\hline MROH5 & 0.9124 \\
\hline MRPL35 & 0.9114 \\
\hline MRPL42 & 0.9217 \\
\hline MRPS25 & 0.9126 \\
\hline MTF1 & 0.9198 \\
\hline MTMR10 & 0.9211 \\
\hline MTMR9 & 0.9197 \\
\hline MTR & 0.9054 \\
\hline MTUS1 & 0.9118 \\
\hline MXD1 & 0.9125 \\
\hline MYLK & 0.9199 \\
\hline MYO18A & 0.92 \\
\hline MYO18B & 0.9225 \\
\hline MYO5C & 0.9172 \\
\hline MYO9A & 0.916 \\
\hline N4BP2 & 0.9176 \\
\hline N4BP2L2 & 0.92 \\
\hline NA & 0.9049 \\
\hline NA & 0.9053 \\
\hline NA & 0.9055 \\
\hline NA & 0.9125 \\
\hline NA & 0.913 \\
\hline NA & 0.9216 \\
\hline NA & 0.9239 \\
\hline NABP1 & 0.9201 \\
\hline NACC2 & 0.9051 \\
\hline NAP1L1 & 0.9215 \\
\hline NCKAP1 & 0.924 \\
\hline $\mathrm{NCOA} 2$ & 0.9122 \\
\hline NDST1 & 0.9171 \\
\hline NDUFA5 & 0.9127 \\
\hline NDUFA9 & 0.9055 \\
\hline NDUFS1 & 0.9133 \\
\hline NEDD4 & 0.9198 \\
\hline NEGR1 & 0.9226 \\
\hline NF1 & 0.9176 \\
\hline NFASC & 0.9215 \\
\hline NFAT5 & 0.9236 \\
\hline NFIA & 0.9233 \\
\hline NFIB & 0.9175 \\
\hline NFIC & 0.9053 \\
\hline NHLRC2 & 0.9055 \\
\hline NIN & 0.9127 \\
\hline
\end{tabular}

\begin{tabular}{|c|c|}
\hline NIPA1 & 0.9197 \\
\hline NKD1 & 0.9174 \\
\hline NKTR & 0.9131 \\
\hline NLGN4X & 0.9116 \\
\hline NOL4L & 0.9049 \\
\hline NOVA1 & 0.9214 \\
\hline NOVA2 & 0.9198 \\
\hline NOX5 & 0.9174 \\
\hline NQO2 & 0.9216 \\
\hline NR6A1 & 0.905 \\
\hline NRDE2 & 0.9227 \\
\hline NRXN3 & 0.9175 \\
\hline NT5DC1 & 0.905 \\
\hline NT5DC3 & 0.9053 \\
\hline NTNG2 & 0.9157 \\
\hline NTPCR & 0.905 \\
\hline NTRK3 & 0.9238 \\
\hline NUCKS1 & 0.9127 \\
\hline NUDCD2 & 0.9051 \\
\hline NUDT3 & 0.92 \\
\hline NUDT4 & 0.9132 \\
\hline NUFIP2 & 0.9198 \\
\hline NUPL1 & 0.9171 \\
\hline ODF2L & 0.9196 \\
\hline OGFRL1 & 0.9176 \\
\hline ONECUT2 & 0.9134 \\
\hline ORAI2 & 0.9134 \\
\hline ORC4 & 0.9049 \\
\hline OSBPL8 & 0.9122 \\
\hline OTUD4 & 0.9115 \\
\hline OTUD7A & 0.9216 \\
\hline OTULIN & 0.9175 \\
\hline PAG1 & 0.9216 \\
\hline PAK3 & 0.9132 \\
\hline PANK3 & 0.9133 \\
\hline PAPD5 & 0.9051 \\
\hline PARD3B & 0.9168 \\
\hline PAX5 & 0.9226 \\
\hline PAXIP1 & 0.9122 \\
\hline PBX1 & 0.9198 \\
\hline PCDH10 & 0.9194 \\
\hline PCDH19 & 0.912 \\
\hline PCDH9 & 0.9239 \\
\hline PCDHA4 & 0.9052 \\
\hline
\end{tabular}

\begin{tabular}{|c|c|}
\hline PCNXL4 & 0.9236 \\
\hline PCYT1B & 0.9122 \\
\hline PDE4B & 0.9098 \\
\hline PDE4DIP & 0.9049 \\
\hline PDE5A & 0.919 \\
\hline PDE7A & 0.915 \\
\hline PDIK1L & 0.9101 \\
\hline PDK1 & 0.9231 \\
\hline PDPR & 0.9174 \\
\hline PDXK & 0.9131 \\
\hline PDZD8 & 0.9166 \\
\hline PEAK1 & 0.9231 \\
\hline PELP1 & 0.9159 \\
\hline PEX11A & 0.9152 \\
\hline PEX26 & 0.9234 \\
\hline PGBD5 & 0.9056 \\
\hline PHACTR1 & 0.9167 \\
\hline PHACTR2 & 0.92 \\
\hline PHC3 & 0.9176 \\
\hline PHEX & 0.9121 \\
\hline PHF3 & 0.9053 \\
\hline PHKG2 & 0.9126 \\
\hline PIGP & 0.9131 \\
\hline PIK3C3 & 0.9173 \\
\hline PIK3CA & 0.9223 \\
\hline PITPNM3 & 0.9048 \\
\hline PLCXD3 & 0.9048 \\
\hline PLEKHA1 & 0.9057 \\
\hline PLEKHA3 & 0.9057 \\
\hline PLEKHA8 & 0.9174 \\
\hline PLEKHG4B & 0.9233 \\
\hline PLEKHM1 & 0.9128 \\
\hline PLLP & 0.9052 \\
\hline PLXNA4 & 0.9199 \\
\hline PNRC2 & 0.9146 \\
\hline POLE & 0.9226 \\
\hline POLR1A & 0.9175 \\
\hline POLR3D & 0.9048 \\
\hline POU2F1 & 0.9178 \\
\hline PPARA & 0.9175 \\
\hline PPIP5K2 & 0.9202 \\
\hline PPM1A & 0.9175 \\
\hline PPM1F & 0.9169 \\
\hline PPP1CB & 0.9166 \\
\hline
\end{tabular}


Analysis of miRNA-Mediated ceRNAs in Gastric Neoplasms

\begin{tabular}{|c|c|}
\hline PPP1R12B & 0.9177 \\
\hline PPP1R13B & 0.9128 \\
\hline PPP2R1B & 0.9188 \\
\hline PPP2R5E & 0.921 \\
\hline PRDM11 & 0.9132 \\
\hline PRDM15 & 0.9226 \\
\hline PRDM16 & 0.9052 \\
\hline PRKAA2 & 0.9054 \\
\hline PRKCA & 0.9132 \\
\hline PRKCB & 0.9052 \\
\hline PRLR & 0.9176 \\
\hline PRPF38A & 0.9122 \\
\hline PRRC2B & 0.9048 \\
\hline PRRG3 & 0.9049 \\
\hline PRTG & 0.9233 \\
\hline PSD3 & 0.92 \\
\hline PSMG4 & 0.9049 \\
\hline PTAR1 & 0.9215 \\
\hline PTBP2 & 0.9133 \\
\hline РTBP3 & 0.905 \\
\hline PTCH1 & 0.9057 \\
\hline PTCHD1 & 0.923 \\
\hline PTEN & 0.9172 \\
\hline PTGER3 & 0.9198 \\
\hline PTK2 & 0.9171 \\
\hline PTPN11 & 0.9125 \\
\hline PTPN14 & 0.923 \\
\hline PTPN23 & 0.9048 \\
\hline PTPRT & 0.9133 \\
\hline PURA & 0.9201 \\
\hline PURB & 0.9054 \\
\hline PVRL1 & 0.9048 \\
\hline PYGO1 & 0.9174 \\
\hline QKI & 0.9226 \\
\hline RAB11FIP2 & 0.9165 \\
\hline RAB11FIP4 & 0.92 \\
\hline RAB15 & 0.9106 \\
\hline RAB21 & 0.9217 \\
\hline RAB3C & 0.9201 \\
\hline RAB3IP & 0.9132 \\
\hline RAB6B & 0.9173 \\
\hline RAD51D & 0.9131 \\
\hline RALY & 0.9052 \\
\hline RAP1A & 0.9206 \\
\hline
\end{tabular}

\begin{tabular}{|c|c|}
\hline RAP1B & 0.9227 \\
\hline RAPGEF1 & 0.9162 \\
\hline RASAL2 & 0.9133 \\
\hline RASGEF1B & 0.9049 \\
\hline RASSF5 & 0.9128 \\
\hline RASSF8 & 0.9166 \\
\hline RBBP4 & 0.9129 \\
\hline RBM25 & 0.9124 \\
\hline RBM28 & 0.9227 \\
\hline RBMS2 & 0.9129 \\
\hline RBMS3 & 0.905 \\
\hline $\mathrm{RC} 3 \mathrm{H} 2$ & 0.9212 \\
\hline REL & 0.9055 \\
\hline REPS1 & 0.9125 \\
\hline REPS2 & 0.913 \\
\hline RET & 0.9116 \\
\hline REV1 & 0.9127 \\
\hline REV3L & 0.9216 \\
\hline RFX7 & 0.92 \\
\hline RGMA & 0.9057 \\
\hline RICTOR & 0.9053 \\
\hline RIF1 & 0.9199 \\
\hline RILPL2 & 0.9051 \\
\hline RIMKLA & 0.9198 \\
\hline RIMS2 & 0.9168 \\
\hline RNF115 & 0.9055 \\
\hline RNF150 & 0.9054 \\
\hline RNF152 & 0.9132 \\
\hline RNF165 & 0.9055 \\
\hline RNF217 & 0.92 \\
\hline RNF24 & 0.9175 \\
\hline RORA & 0.9133 \\
\hline RORB & 0.9175 \\
\hline RPAP2 & 0.9217 \\
\hline RPS6KA5 & 0.9241 \\
\hline RPS6KB1 & 0.9118 \\
\hline RRP15 & 0.9199 \\
\hline $\begin{array}{l}\text { RTEL1- } \\
\text { TNFRSF6B }\end{array}$ & 0.9118 \\
\hline RTKN2 & 0.9049 \\
\hline RUNX1T1 & 0.9126 \\
\hline S100A7A & 0.9049 \\
\hline SAMD12 & 0.9131 \\
\hline SAR1A & 0.9052 \\
\hline SARM1 & 0.9201 \\
\hline
\end{tabular}

\begin{tabular}{|c|c|}
\hline SCAI & 0.9176 \\
\hline SCN3B & 0.9048 \\
\hline SCN8A & 0.9209 \\
\hline SCO1 & 0.9056 \\
\hline $\mathrm{SCOC}$ & 0.9119 \\
\hline SCUBE1 & 0.9132 \\
\hline SDHC & 0.9057 \\
\hline SDK2 & 0.905 \\
\hline SDR42E1 & 0.9216 \\
\hline SEC22C & 0.9049 \\
\hline SEMA3A & 0.9051 \\
\hline SEMA5A & 0.9133 \\
\hline SEMA6D & 0.9169 \\
\hline SERINC3 & 0.9116 \\
\hline SERINC5 & 0.9052 \\
\hline SESN2 & 0.9096 \\
\hline SESN3 & 0.9225 \\
\hline $\begin{array}{l}\text { SF3B3 } \\
\end{array}$ & 0.9053 \\
\hline SGCD & 0.9199 \\
\hline SH3BP2 & 0.9056 \\
\hline SH3PXD2A & 0.9215 \\
\hline SH3TC2 & 0.9132 \\
\hline SHE & 0.913 \\
\hline SHPRH & 0.9216 \\
\hline SHROOM4 & 0.9198 \\
\hline SIK2 & 0.9175 \\
\hline SIK3 & 0.9209 \\
\hline SIM1 & 0.9129 \\
\hline SIX4 & 0.9122 \\
\hline SKP1 & 0.9216 \\
\hline SLC16A7 & 0.9133 \\
\hline SLC1A2 & 0.9201 \\
\hline SLC24A4 & 0.9214 \\
\hline SLC30A4 & 0.9052 \\
\hline SLC30A9 & 0.9126 \\
\hline SLC35B4 & 0.9194 \\
\hline SLC35C2 & 0.9129 \\
\hline SLC35E3 & 0.9217 \\
\hline SLC39A9 & 0.9122 \\
\hline SLC43A2 & 0.9214 \\
\hline SLC44A1 & 0.9132 \\
\hline SLC4A4 & 0.912 \\
\hline SLC4A7 & 0.9104 \\
\hline SLC4A8 & 0.9056 \\
\hline
\end{tabular}


Analysis of miRNA-Mediated ceRNAs in Gastric Neoplasms

\begin{tabular}{|c|c|}
\hline SLC5A3 & 0.9177 \\
\hline SLC7A11 & 0.9133 \\
\hline SLC7A14 & 0.9172 \\
\hline SLC7A2 & 0.9048 \\
\hline SLC7A6 & 0.905 \\
\hline SLC8A1 & 0.9236 \\
\hline SLCO5A1 & 0.905 \\
\hline SLITRK5 & 0.9238 \\
\hline SMAD2 & 0.9241 \\
\hline SMAD5 & 0.9225 \\
\hline SMC1A & 0.9198 \\
\hline SMG9 & 0.9121 \\
\hline SMURF2 & 0.9131 \\
\hline SNAP91 & 0.9125 \\
\hline SNTB2 & 0.9199 \\
\hline SNX1 & 0.9133 \\
\hline SNX27 & 0.9173 \\
\hline SNX30 & 0.9054 \\
\hline SNX33 & 0.913 \\
\hline SNX8 & 0.9191 \\
\hline SOD2 & 0.9134 \\
\hline SOGA3 KIAA0408 & 0.9055 \\
\hline SORT1 & 0.9128 \\
\hline SOS1 & 0.9194 \\
\hline SOX5 & 0.9122 \\
\hline SP3 & 0.9115 \\
\hline SPATA2 & 0.911 \\
\hline SPEF2 & 0.9097 \\
\hline SPRY3 & 0.9131 \\
\hline SREK1IP1 & 0.9131 \\
\hline SRGAP1 & 0.9237 \\
\hline SRRM4 & 0.9172 \\
\hline SSBP2 & 0.9132 \\
\hline $\mathrm{SSH} 2$ & 0.9124 \\
\hline SSTR2 & 0.9052 \\
\hline ST6GALNAC3 & 0.9124 \\
\hline ST8SIA1 & 0.9132 \\
\hline ST8SIA3 & 0.9176 \\
\hline ST8SIA5 & 0.9057 \\
\hline STAM2 & 0.9116 \\
\hline STARD8 & 0.9197 \\
\hline STK24 & 0.9216 \\
\hline STK35 & 0.9212 \\
\hline STOX2 & 0.913 \\
\hline
\end{tabular}

\begin{tabular}{|c|c|}
\hline STRN & 0.9049 \\
\hline STX7 & 0.9217 \\
\hline STXBP4 & 0.9134 \\
\hline STXBP6 & 0.9155 \\
\hline SUGT1 & 0.9202 \\
\hline SULT1B1 & 0.905 \\
\hline SV2B & 0.9228 \\
\hline SV2C & 0.9176 \\
\hline SYK & 0.9121 \\
\hline SYNE3 & 0.9216 \\
\hline SYNJ1 & 0.9119 \\
\hline SYT14 & 0.9134 \\
\hline SYT16 & 0.9134 \\
\hline TAB3 & 0.9052 \\
\hline TACR3 & 0.9166 \\
\hline TBC1D15 & 0.9119 \\
\hline TBC1D16 & 0.9201 \\
\hline TBC1D32 & 0.9173 \\
\hline TBC1D5 & 0.9121 \\
\hline TBX18 & 0.9169 \\
\hline TCF4 & 0.913 \\
\hline TET2 & 0.9198 \\
\hline TET3 & 0.913 \\
\hline TEX14 & 0.905 \\
\hline TFDP2 & 0.9104 \\
\hline TFEC & 0.9195 \\
\hline TG & 0.9195 \\
\hline TGFBR3 & 0.913 \\
\hline THBS1 & 0.9124 \\
\hline THRB & 0.9051 \\
\hline THSD7A & 0.9168 \\
\hline THUMPD3 & 0.9101 \\
\hline TMED3 & 0.924 \\
\hline TMED5 & 0.9171 \\
\hline TMED7 & 0.9181 \\
\hline TMEM120B & 0.9053 \\
\hline TMEM127 & 0.913 \\
\hline TMEM132B & 0.9174 \\
\hline TMEM154 & 0.9235 \\
\hline TMEM164 & 0.9196 \\
\hline TMEM168 & 0.9049 \\
\hline TMEM170A & 0.921 \\
\hline TMEM170B & 0.9133 \\
\hline TMEM178B & 0.9177 \\
\hline
\end{tabular}

\begin{tabular}{|c|c|}
\hline TMEM184A & 0.9052 \\
\hline TMEM192 & 0.9176 \\
\hline TMEM200C & 0.9052 \\
\hline TMOD1 & 0.9101 \\
\hline TMOD2 & 0.9132 \\
\hline TMOD3 & 0.92 \\
\hline TNFAIP8 & 0.92 \\
\hline TNKS1BP1 & 0.921 \\
\hline TNPO1 & 0.9053 \\
\hline TNRC6A & 0.9226 \\
\hline TNRC6B & 0.9134 \\
\hline TOM1L2 & 0.9194 \\
\hline TPCN1 & 0.9116 \\
\hline TPPP & 0.9052 \\
\hline TREM1 & 0.909 \\
\hline TRHDE & 0.9234 \\
\hline TRIL & 0.9102 \\
\hline TRIM33 & 0.9168 \\
\hline TRIM44 & 0.9227 \\
\hline TRIOBP & 0.9237 \\
\hline TRMT5 & 0.9122 \\
\hline TROVE2 & 0.9199 \\
\hline TRPM3 & 0.9129 \\
\hline TRPS1 & 0.9127 \\
\hline TSC1 & 0.9053 \\
\hline $\mathrm{TSC} 2$ & 0.9051 \\
\hline TSC22D2 & 0.9177 \\
\hline TSPAN14 & 0.9231 \\
\hline TSPAN3 & 0.9129 \\
\hline TTBK2 & 0.9173 \\
\hline ТTC39B & 0.9176 \\
\hline TTC7B & 0.924 \\
\hline TTL & 0.9057 \\
\hline TTPAL & 0.9128 \\
\hline TXLNG & 0.9108 \\
\hline TXNDC15 & 0.9115 \\
\hline TXNL1 & 0.9128 \\
\hline UBA6 & 0.9051 \\
\hline UBN2 & 0.9238 \\
\hline UBXN10 & 0.9048 \\
\hline UBXN7 & 0.9132 \\
\hline UFM1 & 0.9151 \\
\hline UHMK1 & 0.9131 \\
\hline UNC119B & 0.9124 \\
\hline
\end{tabular}


Analysis of miRNA-Mediated ceRNAs in Gastric Neoplasms

\begin{tabular}{|c|c|}
\hline UNC13A & 0.905 \\
\hline USP15 & 0.9215 \\
\hline USP31 & 0.9174 \\
\hline USP35 & 0.9163 \\
\hline USP38 & 0.9052 \\
\hline USP42 & 0.9049 \\
\hline USP45 & 0.905 \\
\hline USP46 & 0.913 \\
\hline USP49 & 0.9054 \\
\hline USP6 & 0.9053 \\
\hline USP6NL & 0.9227 \\
\hline USP8 & 0.9231 \\
\hline UVSSA & 0.9126 \\
\hline VAMP4 & 0.9049 \\
\hline VANGL1 & 0.9176 \\
\hline VAPA & 0.9199 \\
\hline VASH2 & 0.9181 \\
\hline VCPIP1 & 0.9173 \\
\hline VGLL3 & 0.9175 \\
\hline VKORC1L1 & 0.9126 \\
\hline VLDLR & 0.9129 \\
\hline VPS35 & 0.9123 \\
\hline VTA1 & 0.9131 \\
\hline VTI1A & 0.9164 \\
\hline VWC2 & 0.9132 \\
\hline WASF3 & 0.9161 \\
\hline WDFY2 & 0.9133 \\
\hline WDR11 & 0.9161 \\
\hline WDR62 & 0.9197 \\
\hline WDR7 & 0.9216 \\
\hline WDR82 & 0.916 \\
\hline WHSC1L1 & 0.9126 \\
\hline WNK3 & 0.9196 \\
\hline
\end{tabular}

\begin{tabular}{|c|c|}
\hline WNT2B & 0.9233 \\
\hline WSCD1 & 0.9118 \\
\hline WTIP & 0.9177 \\
\hline XIAP & 0.9174 \\
\hline XKR4 & 0.9234 \\
\hline XPO1 & 0.917 \\
\hline XPO4 & 0.9225 \\
\hline XYLT1 & 0.9131 \\
\hline YIPF4 & 0.9217 \\
\hline YIPF6 & 0.9051 \\
\hline YOD1 & 0.9198 \\
\hline YY1 & 0.913 \\
\hline ZADH2 & 0.9131 \\
\hline ZBED3 & 0.9126 \\
\hline ZBTB25 & 0.9215 \\
\hline ZBTB34 & 0.9195 \\
\hline ZBTB37 & 0.9239 \\
\hline ZBTB44 & 0.9053 \\
\hline ZBTB8A & 0.9049 \\
\hline ZВТВ8B & 0.9202 \\
\hline $\mathrm{ZC} 3 \mathrm{H} 12 \mathrm{C}$ & 0.9172 \\
\hline $\mathrm{ZC} 3 \mathrm{H} 14$ & 0.9239 \\
\hline ZC3H6 & 0.9215 \\
\hline ZC3H8 & 0.917 \\
\hline ZDHHC17 & 0.9183 \\
\hline ZDHHC18 & 0.9121 \\
\hline ZDHHC2 & 0.9127 \\
\hline ZDHHC21 & 0.9215 \\
\hline ZEB1 & 0.9232 \\
\hline ZFHX4 & 0.9114 \\
\hline ZFP90 & 0.9127 \\
\hline ZFYVE20 & 0.9167 \\
\hline ZFYVE26 & 0.9054 \\
\hline
\end{tabular}

\begin{tabular}{ll}
\hline ZHX3 & 0.9175 \\
\hline ZNF107 & 0.9164 \\
\hline ZNF117 & 0.9238 \\
\hline ZNF138 & 0.9226 \\
\hline ZNF142 & 0.913 \\
\hline ZNF189 & 0.9114 \\
\hline ZNF207 & 0.923 \\
\hline ZNF223 & 0.9106 \\
\hline ZNF226 & 0.9231 \\
\hline ZNF230 & 0.9125 \\
\hline ZNF233 & 0.9182 \\
\hline ZNF257 & 0.9232 \\
\hline ZNF26 & 0.9234 \\
\hline ZNF268 & 0.9057 \\
\hline ZNF273 & 0.9227 \\
\hline ZNF286A & 0.9128 \\
\hline ZNF286B & 0.9127 \\
\hline ZNF292 & 0.9111 \\
\hline ZNF37A & 0.9131 \\
\hline ZNF431 & 0.9177 \\
\hline ZNF445 & 0.9176 \\
\hline
\end{tabular}

Supplementary 2: List of genes containing T-UCR in their exonic regions according to the study of Bejerano et al.

\begin{tabular}{lll} 
uc.143 & 218 & AB014560 \\
\hline uc.203 & 203 & AB067798 \\
\hline uc.135 & 201 & AK096400 \\
\hline uc.339 & 252 & ATP5G2 \\
\hline uc.413 & 272 & BC060758 \\
\hline uc.49 & 207 & BC060860 \\
\hline uc.61 & 326 & BCL11A \\
\hline uc.324 & 225 & C11orf8 \\
\hline uc.285 & 232 & CARP-1 \\
\hline uc.233 & 266 & CENTG3 \\
\hline
\end{tabular}

\begin{tabular}{lll} 
uc.393 & $\mathbf{2 7 5}$ & CLK3 \\
\hline uc.185 & 411 & CLK4 \\
\hline uc.184 & 230 & CPEB4 \\
\hline uc.471 & 239 & DDX3X \\
\hline uc.331 & 218 & DLG2 \\
\hline uc.13 & 237 & EIF2C1 \\
\hline uc.194 & 201 & EPHA7 \\
\hline uc.183 & 236 & FBXW1B \\
\hline uc.333 & 270 & FLJ25530 \\
\hline uc.478 & 252 & GRIA3 \\
\hline
\end{tabular}




\begin{tabular}{|c|c|c|c|c|c|}
\hline uc. 479 & 302 & GRIA3 & uc. 77 & 296 & ZFHX1B \\
\hline uc. 282 & 207 & GRIN1 & uc. 151 & 214 & ZFR \\
\hline uc.97 & 442 & HAT1 & uc. 474 & 210 & ZNF261 \\
\hline
\end{tabular}

\section{Discussion}

Gastric neoplasm is the leading cause of cancerrelated deaths. According to research conducted in 2008, gastric neoplasm is the fourth most common cancer in the world and ranks second among cancers that cause death. The death rate from this cancer is higher than that from malignant tumors such as colon, breast and prostate cancers. The development of this cancer is complex, involving a number of genetic and epigenetic alterations of oncogenes, tumor suppressor genes, deoxyribonucleic acid (DNA) repair genes, cell cycle regulators, and signaling molecules. Oncogenes are activated at different stages of the course of gastric neoplasm, and some tumor suppressor genes are inactivated. Numerous studies have shown that miRNAs can be effective in carcinogenesis. Changes in expression levels of miRNAs in different types of cancer have been investigated, and miRNAs have been observed to differ between normal and pathological tissues (Sevignani et al., 2006; Zhou et al., 2010). Various miRNAs have been shown to play a specific role in tumor progression and metastasis in the differentiation of cancer cells (Kim et al., 2011; Calin et al., 2002; Michael et al., 2003; Metzler et al., 2004; Chan et al., 2005; He et al., 2005; Sevli et al., 2010; Lamy et al., 2006; Iorio et al., 2005) The purpose of this study was to describe novel biomarkers for GN through in silico analysis involving gastric neoplasm-specific miRNAs, by determining their combinatorial target genes (potential ceRNAs), selecting those with T-UCR and potentiating their association with gastric neoplasm using statistical correlation techniques.

Four miRNAs experimentally related to gastric neoplasm were identified through the miRTarbase database (Table I). Genes with equal ComiR abundance were listed through 1008 genes targeted concurrently by these four miRNAs. Genes with TUCR in their exonic regions were described from the genes containing T-UCR listed by Bejerano et al. (Bejerano et al., 2004). We then considered those exhibiting probable ceRNA activity in our earlier analysis (Table II). Next, we choosed genes with significant differences in expression between gastric neoplasm and normal gastric tissues from GN-related ceRNAs involving T-UCR. This test revealed significantly higher NFAT5 expression in gastric neoplasm than in normal stomach tissue, 
while the other exhibited no significantly different expression pattern. In addition, the NFAT5 and CLK3 gene pair were substantively associated with gastric neoplasm based on the Spearman correlation analysis findings.

These NFAT5 genes have not previously been experimentally linked to gastric neoplasm. Ours is the first study to associate these two genes with gastric neoplasm. NFAT family contains five different proteins one of them is NFAT5 protein. But, NFAT1 to 4 proteins are regulated by calcineurin, NFAT5 is controlled by osmotic pressure at the nuclear localization, transcriptional and expression levels. When stimulated, NFAT5 triggers target gene transcription by binding to tonicity enhancer elements) in various coordinator domains which are all responsible for supplying cells in order to facilitate their survival under hypertonic conditions (Cheung and Ko, 2017). NFAT5 gene shows its oncogenic role via different pathways in such diseases as renal cell carcinoma, breast cancer, lung adenocarcinoma and colon cancer. NFAT5-related expression of S100A4 projects the migration and proliferation of renal carcinoma cells (Küper et al., 2014). Additionally, NFAT5/STAT3 interaction soften synergism of high salt with IL-17 towards induction of VEGF-A expression in breast cancer cells (Amara $\mathrm{S}$ et al., 2016). NFAT5 also stimulates the migration and proliferation of pulmonary adenocarcinoma cells, in part by modulating AQP5 expression (Guo and Jin, 2015). The Src kinase pathway is also involved in NFAT5-mediated S100A4 induction through hyperosmotic stress in colon cancer cells (Chen et al., 2011). NFAT5 is also a tumor suppressor that functions by suppressing invasion and triggering apoptosis in hepatocellular carcinoma.

\section{Conclusion}

The NFAT5 gene was correlated with gastric neoplasm in our study, and in silico analysis results predict that they may potentially play an oncogenic role in gastric neoplasm. The inconsistent results concerning their roles in varying forms of cancer suggests that our study findings will be preliminary for subsequent in vitro and in vivo studies performed to determine the roles of the NFAT5 gene in gastric neoplasm progression. Fatal one among urological cancers. RCC is causedby the accumulation of many genetic and

\section{Ethics Committee Approval:}

Since it is a in silico study, there is no need for an ethics committee approval

Peer-review: Externally peer-reviewed.

Author Contributions: Externally peer-reviewed. Author Contributions: Concept- D.U.A.; Design D.U.A., S.E.; Supervision-D.U.A., S.E.; Materials D.U.A., S.E.; Data Collection and/or Processing D.U.A., S.E.; Analysis and/or InterpretationD.U.A; Literature Review-D.U.A.; WritingD.U.A.; Critical Review- S.E

Conflict of Interest: No conflict of interest was declared by the author.

Financial Disclosure: The authors declared that this study hasn't received no financial support.

\section{References}

Amara S, Alotaibi D, Tiriveedhi V. NFAT5/STAT3 interaction mediates synergism of high salt with IL-17 towards induction of VEGF-A expression in breast cancer cells. Oncol Lett. 2016; 12(2):933-43.

Bejerano G, Pheasant M, Makunin I. Ultraconserved Elements in the Human Genome. Science. 2004; 304(5675), pp. 13211325

Calin GA, Dumitru CD, Shimizu M. Frequent deletions and down-regulation of micro- RNA genes miR15 and miR16 at 13q14 in chronic lymphocytic leukemia. Proc Natl Acad Sci. 2002; 99(24):15524-9.

Chan JA, Krichevsky AM, Kosik KS. MicroRNA21 is an antiapoptotic factor in human glioblastoma cells. Cancer Res. 2005; 65:6029

Cheung CY, Ko BC. NFAT5 in cellular adaptation to hypertonic stress-regulations and functional significance. J Mol Signal. 2013; 8(1):5.

Chen M, Sastry SK, O'Connor KL. Src kinase pathway is involved in NFAT5-mediated S100A4 induction by hyperosmotic stress in colon cancer cells. Am J Physiol-Cell Ph. 2011; 300(5):C1155-C63.

Chou CH, Shrestha S, Yang, CD. miRTarBase update 2018: a resource for experimentally validated microRNA-target interactions Nucleic Acids Research, 2018; 46 (D1), D296-D302,

Coronnello C, Benos PV. ComiR: combinatorial microRNA target prediction tool. Nucleic acids research. 2013; 41(W1), W159-W164. 
Fassan M, Dall'Olmo L, Galasso M, Braconi C, Pizzi M, Realdon S, Volinia S, Valeri $\mathrm{N}$, Gasparini P, Baffa R, Souza RF, Vicentini $C$, D'Angelo E, Bornschein $\mathrm{J}$, Transcribed ultraconserved noncoding RNAs (TUCR)are involved in Barrett's esophaguscarc inogenesis.Onco Target.2014: 30;5(16):716271.

Guo K, Jin F. NFAT5 promotes proliferation and migration of lung adenocarcinoma cells in part through regulating AQP5 expression. Biochem Bioph Res Co. 2015; 465(3):644-9.

He H, Jazdzewski K, Li W, Liyanorachchi S, Nagy $\mathrm{R}$, Volinia $\mathrm{S}$. The role of microRNA genes in papillary thyroid carcinoma. Proc Natl Acad Sci USA 2005; 102:19075-80.

Iorio MV, Ferracin M, Liu C, Veronese A, Spizzo R, Sabbioni S. MicroRNA gene expression deregulation in human breast cancer. Cancer Research 2005; 65:7065-70.

Kim YK, Yeo J, Ha M. Cell adhesion- dependent control of microRNA decay. Molecular Cell 2011; 43:1005-14.

Küper C, Beck F-X, Neuhofer W. NFAT5mediated expression of S100A4 contributes to proliferation and migration of renal carcinoma cells. Front Physiol. 2014; 5:293

Lamy P, Andersen CL, Dyrskjøt L, Tørring N, Ørntoft T, Wiuf C. Are microRNAs located in genomic regions associated with cancer? $\mathrm{Br} \mathrm{J}$ Cancer 2006; 95 (10): 1415-18

Metzler M, Wilda M, Busch K. High expression of precursor microRNA-155/BIC RNA in children with Burkitt lymphoma. Genes Chromosomes Cancer. 2004; 39:167-9.

Michael MZ, O.Connor SM, van Holst Pellekaan NG. Reduced accumulation of specific microRNAs in colorectal neoplasia. Mol Cancer Res. 2003; 1:882-91.

Pan H.W, Li S.C, Tsai K.W. MicroRNA Dysregulation in Gastric Cancer, Current Pharmaceutical Design, 2013; 19(7):1273-84.

Qi X, Zhang D.H, Wu N, Xiao J.H, Wang X, Ma W. CeRNA in cancer: possible functions and clinical implications. $\mathrm{J}$ Med Genet 2015; 0:1-9. doi:10.1136/jmedgenet2015-103334.

Sevignani C, Calin GA, Siracusa LD. Mammalian microRNAs: a small world for fine-tuning gene expression. Mamm Genome. 2006; 17(3):189202.
Sevli S, Uzumcu A, Solak M, Ittman M, Ozen M. The function microRNAs, small potent molecules in human prostate cancer. Prostate Cancer P D 2010; 13:208-17.

Schiller MP, Wilkerson PM. Gastric neoplasms. Surgery. 2017: 35:(11): 635-643

Tang Z, Li C, Kang B. GEPIA: a web server for cancer and normal gene expression profiling and interactive analyses. Nucleic acids research. 2017; 45(W1), W98-W102.

Zhou YM, Chen LJ, Barlogie B. High-risk myeloma is associated with global elevation of miRNAs and overexpression of EIF2C2/AGO2. Proceedings of the National Academy of Sciences of the United States of America. 2010; 107: 7904-9. 